\title{
CLINICAL MANIFESTATIONS OF PATIENTS WITH PRIMARY SJÖGREN'S SYNDROME
}

\author{
Vítor de Castro Grotti1,*, Alexandra Estrella Lisoni², Gabriela Guilhoto Cabral Lamonica², Waldenise Cossermelli² \\ 1.Hospital de Caridade São Vicente de Paulo, Jundiaí (SP), Brazil; 2.Faculdade de Medicina de Jundiaí, Jundiaí (SP), Brazil. \\ *Corresponding author: vitor.grotti@hotmail.com
}

\section{BACKGROUND}

Sjögren's syndrome (SS) is a chronic autoimmune condition known by its effects on exocrine glands due to lymphocytic infiltration, leading to sicca symptoms. Besides, systemic or extraglandular manifestations can also appear in these patients, apart from an increased risk of developing non-Hodgkin's B-cell lymphoma. The most affected group is middle aged women and its clinical diagnosis is based on objective findings of ocular and oral dryness or glandular parenchymal damage, along with serological criteria or glandular histopathologic evidence of autoimmunity. Considering that the disease has a broad range of severity and clinical features, the study aims to analyze the spectrum of the disease in order to recognize the main symptoms in this population.

\section{METHODS}

The medical records of patients were selected based on the confirmed diagnosis of SS, excluding patients with associated rheumatologic diseases, which, as seen in the literature, correspond to about $60 \%$ of the cases. In total, 38 women were diagnosed with SS and only 17 had the primary form of the disease. The diagnosis meet the international criteria and the symptoms were evaluated through clinical examination, laboratory findings and dosage of autoantibodies.

\section{RESULTS}

In the group of patients, the mean age was 54 , of which $17.6 \%$ were young (between 18 and 41 years) and $82.3 \%$ were middle age (from 42 to 66 years). Among the patients studied, clinical manifestations were disposed in the following descending order: xerophthalmia (100\%), dry mouth (94\%), musculoskeletal (53\%), fatigue (47\%), dry skin (35\%), peripheral neuropathy (35\%), vasculitis (17\%), Raynaud's (12\%), lymphoma (5\%) and others (17\%). Furthermore, anti-SSA and anti-SSB were detected in 76 and $47 \%$ of subjects, respectively.

\section{CONCLUSION}

By analyzing the disease clinical data, we can observe that the main symptoms are those from the glandular spectrum, as seen in our patients. However, contrary to what is seen in the literature, peripheral neuropathy ( $35 \mathrm{vs.} 22 \%$ ) and vasculitis (17vs. $12 \%$ ) were more prevalent in our study, whereas Raynaud's (12 vs. 30\%) and dry skin ( 35 vs. 55\%) were less seen. As for autoantibodies, the prevalence of anti-SSA and anti-SSB follows what is expected for the disease, as well as the risk of progression to lymphoma. We can conclude that although the clinical manifestations are well established, they can variate depending on the region and population, reinforcing the importance of early diagnosis of the most prevalent complications and its management.

\section{KEYWORDS}

Sjögren's syndrome, Neuropathy, Vasculitis, Clinical manifestations. 